\title{
心理咨询理论与实践
}

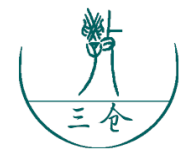

sciscan

\section{一例系统式家庭治疗方法在幻咱 倾向咨询中运用的案例报告}

\author{
王晓霞 \\ 山西财经大学, 太原, 030006 \\ 邮箱: wxx2211@163.com
}

摘 要：系统式家庭治疗是运用系统式思维方法, 把关注的焦点置于人际关系 上的心理治疗范式。主张实施心理干预的对象应当是在关系和系统上，而不仅 仅是个体。本案例利用系统式家庭治疗方法, 对一名幻嗅倾向大学生进行了心 理咨询，从分析家庭系统结构的角度理解个体呈现的问题，通过家庭系统重构 对来访者进行治疗。

关键词：系统式思维；系统式家庭治疗；心理咨询

收稿日期：2019-06-09; 录用时间：2019-07-11；发表时间：2019-07-20

\section{A Case Report of Systematic Family Therapy Applied in Psychedelic Odor Tendency Counseling}

\author{
Wang Xiaoxia
}

Shanxi University of Finance and Economics, Taiyuan 030006

Abstract: Systematic family therapy is a psychotherapy paradigm that focuses on 
interpersonal relationships by using systematic thinking method. The object of psychological intervention should be relationship and system, not only individual. This case uses systematic family therapy to give psychological counseling to a college student with hallucinatory tendency. From the perspective of analyzing the structure of family system, this case understands the problems of individual presentation and treats the visitors through the reconstruction of family system.

Key words: Systematic thinking; Systematic family therapy; Counseling

Received: 2019-06-09; Accepted: 2019-07-11; Published: 2019-07-20

Copyright () 2019 by author(s) and SciScan Publishing Limited

This article is licensed under a Creative Commons Attribution-NonCommercial 4.0 International License.

https://creativecommons.org/licenses/by-nc/4.0/

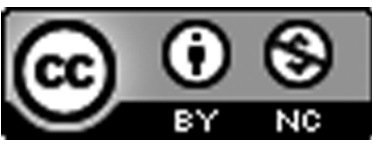

\section{1 背景}

来访者, 女, 大二在校学生。第一次来访原因是，总感到自己身体有味道， 怕别人闻到，不敢和人靠近。在宿舍和教室都想办法远离别人。和人靠近时有 焦虑情绪出现，每当和人靠近时会僵硬，不自然，无法正常交流，因此问题影 响人际关系，很苦恼。

来访者初次发生这个问题是在高二的时候，当时有同学说在教室闻到臭味， 后来有同学说闻到她身上散发出了臭味。来访者开始在意自己身上的味道，总 感觉自己身上有味道，从而不敢和人靠近。希望达到的治疗目标：消除这些紧 张情绪，能够和人坐在一起正常交流。至今咨询了六次，并且还在继续。 


\section{2 评估与分析}

\section{1 案例的评估和诊断}

对于这一案例，首先对来访者进行了评估和诊断。根据病与非病三原则： 主客观世界的统一、精神活动内在协调性以及个性相对稳定性原则，通过访谈， 咨询师认为来访者现实感尚存, 存在一定的嗅觉幻想。精神活动内在协调性较 好, 个性稳定。存在一定程度的焦虑和紧张情绪。来访者认知功能、抽象功能、 整合功能、自我保存功能基本完好，生活基本没有被干扰，比较正常，可以进 行心理咨询和治疗。

\section{2 咨询方法与适用原理}

家庭治疗模式开始于 20 世纪 50 年代的美国。其创始人 Nathan Ackerman 最 初是用这一模式来解决当时美国社会出现的大量的家庭离异、重组和青少年犯 罪的问题。1997-1999 年德中心理治疗研究院开展的培训项目，促进家庭治疗 模式在中国的传播。家庭治疗是一种把关注的焦点置于人际关系上的心理治疗 范式。它认为, 个体只有在互动和系统 (家庭) 中才能被说明、被理解，个体呈 现的问题实际上反映个体所在的系统出现了问题，因此，实施心理干预的对象 应当是在关系和系统上，而不仅仅是个体 [1]。家庭是个体活动的主要场所, 是 精神应激和社会支持的重要来源。家庭的人际互动与成员的心理状况相互影响， 良好的人际互动与良好的心理健康关联, 个人的心理健康问题也可以看作是家庭 系统影响的结果 [2][3]。系统家庭治疗模式的重点在于围绕症状找出家庭规则和 家庭沟通模式中的问题，加以 “扰动”, 从而促成症状的消失。

\section{3 目标与计划}

在初始访谈中，咨询师使用了一定的情境化方法，了解来访者症状的背景 和情境。进而与来访者明确诉求、签订契约、确立治疗目标和目的。例如向来 访者明确是谁有咨询愿望的? 想要得到什么样的帮助? 希望解决什么问题? 怎 样才能解决问题? 对咨询的期待是什么? 
当然咨询目标的确定并不是一蹴而就的，可能贯穿于整个咨询过程中。我 们会在整个咨询中不断地明确来访者的咨询目标。对于此来访者, 咨询师认为 可以用系统式家庭治疗方式来进行咨询治疗。预计咨询次数为十次。预计达到 的目标是, 对来访者及其家庭进行一些扰动, 从而起到改变来访者固化视角, 减轻其症状的作用。

\section{4 过程}

第二次咨询中，咨询师用循环提问的方式与来访者进行对话。当问到问题 例外的时候咨询师问道：“问题会在什么时候消失或者减轻呢? 在和什么人在 一起的时候没有这种问题呢?” 来访者回答：“问题在和熟悉的好朋友在一起 的时候会比较好。这时我和他们可以离得近, 交谈也比较自然。另外在图书馆 学习的时候也会比较好。和别的班级陌生的同学在一起也会好一些。”因此咨 询师推论, 来访者和宿舍、本班级同学在一起时问题会明显, 也就是和相互认识, 但又不太熟悉的人在一起时问题会明显一些。从这一点我们可以假设来访者在 本来应该是重要人际的圈子里，担心打不好交道，会出现问题。担心自己在重 要他人心目中的位置。另一个假设是，来访者似乎渴求亲密关系，又要远离回 避亲密关系。

我们在第三次咨询时画了家谱图。来访者的家庭关系以及家谱图（见图 1)。

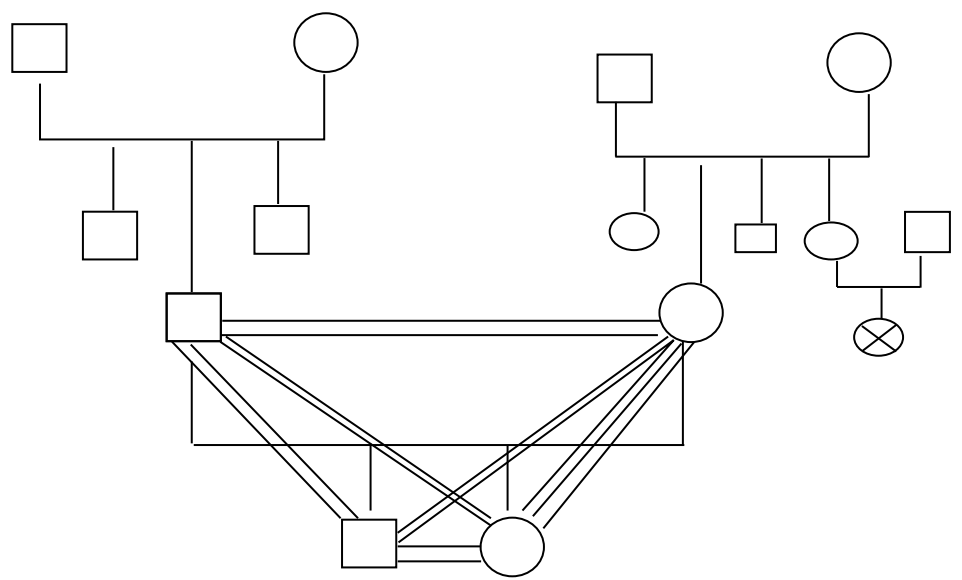

图 1 来访者家谱图

Figure 1 The Family Tree of the Client 
来访者和爸爸、妈妈、哥哥生活在一起。有一个大七岁的哥哥。但是了解 信息时，来访者对哥哥的年龄记忆很模糊，说不清哥哥到底几岁，对小时候和 哥哥在一起的记忆也很模糊。和哥哥关系比较疏远, 不愿意长时间和哥哥在一起， 感觉哥哥不能对她太好，例如给她钱。如果哥哥对她好，有要把哥哥推开的感觉。 但是说内心还是很喜欢哥哥的。来访者和妈妈的关系密切，无话不谈，用来访 者的话说，妈妈是无原则溺爱。值得注意的是，来访者和妈妈一直睡同一张床。 现在放假回家仍然会和妈妈睡同一张床，爸爸睡另外一个房间。妈妈和爸爸的 关系不错，但有一个现象，妈妈在家的时候，爸爸总是出去闲逛不回家。但是 妈妈出去工作的时候，爸爸又会打电话跟踪，还会和女儿说怀疑妈妈有外遇。 来访者的爸爸是在姑姑家寄养长大的。和两个兄弟都不亲近。母亲这边, 姐姐 大很多。一个弟弟没有结婚。妹妹的女儿在初二的时候去世了。爷爷奶奶都去 世了，姥爷去世了，姥姥在世。

通过家谱图我们从整个家庭的宏观角度，对家庭进行了新的审视，也重新 收集到了很多以前通过问答无法收集到的信息。通过家谱图，来访者从新的视 角看到了家庭中的一些关系，对其起到了一定的扰动作用。例如看到爸爸寄养 经历对其性格的影响，爸爸和妈妈若即若离关系的形成原因的重新构建等。

第四次来访者来的时候说本周挺开心的，但是同时也会感到自己不能忘掉 身上有味道的事情，不能变得那么开心。因此会主动想起这件事，重新回到郁 郁寡欢的状态。可见，来访者不能也不愿意摆脱这个问题，这个问题给她带来 了一定的意义，具有症状的功能性。当咨询师问道：“妈妈知道你不开心吗? 她对于你的这个问题是怎样的看法? 有什么反应? ” 来访者说： “妈妈并不以 为然, 觉得这并不是个问题。”这让她感到父母都不关心自己，也不理解自己。 并且每次和妈妈打电话，妈妈都会说哥哥结婚找对象的事，这更让她感到妈妈 不关心自己，感到心烦。咨询师问什么情况下这种心情会得到缓解呢? 来访者 回答在和爸爸妈妈在一起的时候会缓解，这时是没有哥哥的。

根据以上所有信息, 咨询师逐渐提出了自己的假设。对此我的假设是, 来 访者希望独占爸爸妈妈的爱，因此排斥哥哥。同时，来访者与母亲的长期同睡， 对其心理发展造成了影响，来访者会形成融合焦虑，在这种分离和融合焦虑的 
双重作用下, 来访者非常矛盾, 因此形成症状。这个症状就是, 感到自己有味道, 希望和他人接近又不敢和他人接近, 从而保持一定距离。这样就满足了和他人 保持距离的愿望, 也就是潜意识中和妈妈分离的愿望。对于来访者说的排泄器 官散发出的味道，咨询师还没有形成假设。

第五次来访者说到妈妈和爸爸的关系。爸爸生过一次病, 疾病后爸爸变得 疑神疑鬼，他一方面远离妈妈，一方面又在妈妈上班的时候打电话追踪。还向 来访者打电话抱怨妈妈出去, 甚至怀疑妈妈有外遇。来访者对于这种情况非常 反感，感到自己很可怜，没有人关心，认为他们没有在情感上关心自己。之后 谈到了男友问题。来访者说有喜欢的男生。但是在对未来的想象中, 感觉自己 是找不到男朋友的。很难想象和一个男性每天在一起, 说自己很可能冷暴力对方。 这些信息更加证实了咨询师对她的假设，她在亲密关系上有问题，有明显的融 合焦虑特征。而这种问题的形成，与她家庭的结构密不可分。父亲的寄养经历， 对其造成的一定程度上的亲密关系问题（和妻子分开焦虑，在一起又要离开）, 妈妈在家庭系统中受到一定的影响。妈妈与女儿的同睡问题，给孩子造成的心 理创伤。女儿与哥哥的竞争、哥哥与母亲的依恋、父母之间的关系等，错综复 杂的家庭系统造成来访者成为家庭、家族问题的展现者。

在第六次咨询中, 来访者提到本周症状有加重的趋势。感觉好像又回来了。 但是与之前的不同是，感觉似乎没有那么绝望了。同时也谈到和家人在一起时 的感觉很糟糕。哥哥总是很消极, 总说自己没有希望, 把希望寄托在妹妹身上。 妈妈压力大, 要操心哥哥的婚事, 女儿也没有毕业, 没有独立。爸爸则是害怕 妈妈离开。用来访者的话说, “爸爸和妈妈之间是病态的好, 我们感觉插不进 去”。爸爸曾经生病。之后就变得像要不到糖的小孩，总是很渴望爱，却又不 知道正常的爱是什么。如果来访者和哥哥不在家, 总会打电话来抱怨自己不舒 服, 回家后他也没事。来访者感到妈妈是一个很有智慧的人, 有很多生活的哲理, 很有能量, 但是最近被压力弄得不如以前了。对于哥哥来访者看不懂，他总会 对妹妹说, “你要好好读书, 多帮帮我” 这样的话。来访者很不愿意听到这样 的话。

之后我们使用了格板。来访者的认为现在他们家庭的状况是这样的, 如图 2: 


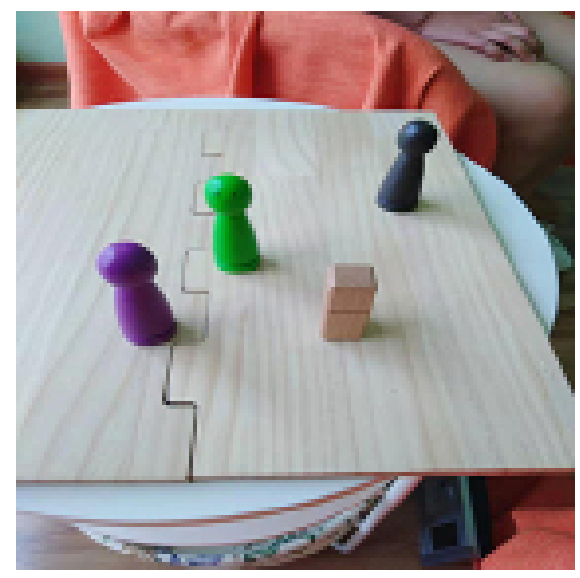

图 2 第一次格盘

Figure 2 The First Family Board

来访者用紫色大人表示妈妈，木头小人表示爸爸。爸爸妈妈面对面，相互 望着。来访者是绿色大人，面向父母站着。她说: “爸爸妈妈两个人关系很紧密， 我感觉插不进去, 又很想加人他们。” 哥哥是一个灰黑色大人。远远地站在那 里望着他们三个。来访者说: “感觉哥哥很抑郁，也很孤独。给我的感受是, 哥哥离家庭很远，也挺孤独，似乎没有人为他考虑。”

这时来访者补充了一些信息，哥哥有一个女友，但却无法结婚。哥哥给出 的原因是，女友家庭条件好，哥哥感觉配不上女友，自己没有钱，没法娶女友。 但是他们分了好几次手，都没有彻底分开。哥哥挺有责任感，没有上过大学， 目前在南方打工。每月给父母和妹妹寄钱。这让咨询师感到，以一个没有学历 的男孩，能够做到给家里寄钱、养家已经很不错了。但是，咨询师又有些好奇， 作为一个三十岁的壮年男性，似乎可以做到更多。可以去学一门技术或者手艺， 可以去做生意，并且在女友家庭条件好，自己感觉配不上女友的情况下，更应 该想办法努力了。可是咨询师并没有感到哥哥为了家庭和女友做出努力，没有 进一步学习，也没有想法提高自己。他似乎在让自己变得无能，给自己设限。

这时咨询师问来访者现在的感受如何，她说其实还可以，就是感觉爸爸妈 妈似乎不关心自己。哥哥也很孤独。我问, 那怎样的改变你会感觉更好呢? 来 访者说变成一个圆，可以互相看到。如图 3。 


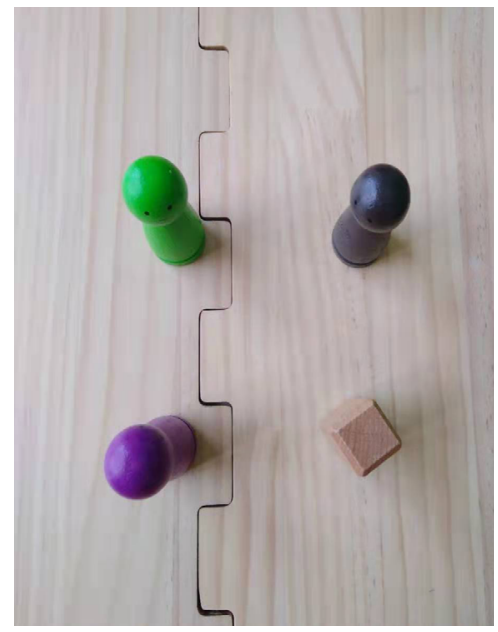

图 3 第二次格盘

\section{Figure 3 The Second Family Board}

咨询师问，如果做出这样的改变，谁应该先动呢? 来访者说，觉得应该爸 爸先动, 爸爸不要老缠着妈妈。这样妈妈就可以看我们了。这时咨询师有个想法, 来访者是否在用身体有味道症状吸引妈妈的注意力。而哥哥也在用不能结婚吸 引妈妈的注意力。这个家庭, 就像是三个嗷嗷待哺的孩子, 在争夺妈妈的注意 力。这时咨询师让来访者找一个木人代表她的问题，一个木人代表哥哥的问题。 摆好后如图 4 所示:

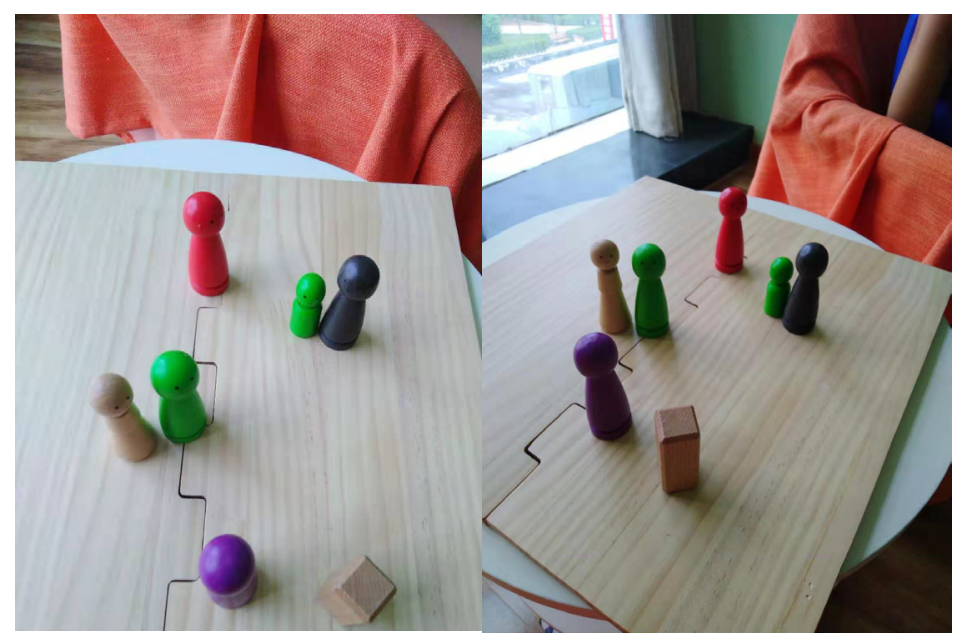

图 4 第三次格盘

Figure 4 The Third Family Board 
来访者选择了一个木色小人代表自己的问题，一个绿色小人代表哥哥的问 题，一个红色小人代表哥哥的女友。咨询师问来访者摆好后的感受，来访者说: 感到自己的问题小人很好看。

从整个改变后的格盘中, 我们看到, 爸爸是一个小小的木头人, 很像一个 小孩子。来访者和问题小人站在一起, 似乎气势更强大了, 引人注目。而哥哥 和问题小人在一起, 本身又是黑黑的颜色，似乎也很引人注意。咨询师问来访者, 这时妈妈什么感受。来访者说感觉妈妈压力挺大。是啊, 咨询师也有同样的感受, 面对三个嗷嘋待哺的孩子, 这个本身很有能量的母亲, 也变成了紫色。这时妈 妈和爸爸不再面对面了, 他们的眼光似乎转向了他们兄妹俩。一家人每个人都 有着自己的问题，似乎又通过各种问题紧密地联系在一起。

这时咨询师在想，改变家庭格局的关键点是什么? 是让爸爸长大吗? 但是 我又感到很难，源于爸爸的寄养经历造成的坚固的人格问题，以及他在生病后 的状态。这时，咨询师想是否可以换一个视角。现在我们看问题解释问题的视 角是问题取向的, 认为妈妈一个人要解决三个人的问题, 妈妈一个人的能量资 源要分给三个人。三个人在嗷嘋待哺。那么, 家庭中其他三个人自身的能量和 资源又哪里去了呢？他们似乎没有看到，忘掉了，也没有利用起来。哥哥一方 面远离家庭, 一方面又用无法结婚、自我设限, 吸引家庭的注意力, 用给家人 生活费和家庭保持着密切的连接。就像是一只要离巢, 又无法离巢的小鸟。妈 妈用催婚保持着他们的连接, 而这种连接又是很有压力的。他们全家是否可以 将每个人的资源都利用起来，共同解决问题，而不是相互施加压力呢? 例如, 妈妈担心儿子的婚姻问题, 是否可以问问 “困难在哪里? 为什么不能结婚? 我 们可以做些什么帮助你解决这个困难? ” 爸爸也可以起到作用，妹妹也可以起 到作用。而不是像现在这样, 都在等待别人的帮助。他们出钱出力出主意都可以, 共同帮助哥哥能够在工作和事业上有所长进，能有资格娶到女友。他们可能忘 掉了自己的力量, 而只记得索取了。

咨询做到这里, 来访者的反馈是, 感到家庭的情况变得更清晰了, 也感受 到了以前没有想到过的看到了以前没有看到的资源，愿意回去试一试。咨询还 没结束，来访者还在继续探索中。 


\section{5 讨论与总结}

这是一则非常有趣的案例，家庭中展现出了非常复杂和深奥的关系，故事 引人人胜。在整个咨询中, 咨询师的咨询方式是, 利用系统式思维方式和家庭 治疗方法，运用家谱图、循环提问、格盘等方式，扰动来访者及其家庭，对来 访者的家庭系统进行探索，对家庭问题进行了重构，并给出了资源取向的重新 解释，从而引导来访者从更多的视角看待自己的家庭，解释自己的问题，起到 改变一点点的作用。但是咨询师在咨询中也感到，这是一个很困难的案例。咨 询师很难撼动如此巨大和有如此多创伤存在的家庭系统。因此, 在学校心理咨 询的背景下, 在咨询师水平非常有限的情况下, 只能做到一点点扰动。改变来 访者的一点点视角，自身也感到这个来访者似乎是很难治愈的，咨询师也需要 督导师们的督导。

\section{参考文献}

[1] 鿊正洪, 赵旭东. 西方家庭治疗的新趋向 $[\mathrm{J}]$. 医学与哲学 (人文社会医学版), 2010, 31 (9) : 35-37.

[2] SARTORIUS N, FALLOON L R H. Research on Family Interventions for Mental Disorders: Problems and Perspectives [ M ] //Families and Mental Disorder: From Burden to Empowerment. Hoboken: John Wiley and Sons, Inc, 2005: 235-259.

[ 3 ] SCHERMERHORN A C, CUMMINGS E M, DAVIES P T. Children' $s$ representations of multiple family relationships: Organizational structure and development in early childhood [ J ] . J Fam Psychol, 2008, 22 ( 1 ) : 89-101. https://doi.org/10.1037/0893-3200.22.1.89

[4] 史靖宇, SCHWEITZER J, 赵旭东. 中德家庭治疗师对系统家庭治疗应用 的评价 $[\mathrm{J}]$. 中国心理卫生杂志，2014，28（1）：15-21.

[5]林红，钱英，王冲，等.中德系统式家庭治疗督导师培训效果 $[\mathrm{J}]$. 中 国心理卫生杂志，2019，33（1）：1-7.

[6]武志伟. 系统家庭治疗模式在个别化青少年中的应用一一基于一项个案的 研究 $[\mathrm{J}]$. 社会工作, 2012（5）：51-53. 